\title{
Kyphoplasty for the Treatment of Vertebral Compression Fractures in a Cancer Patient with Neurological Deficits and Anterior Vertebral Wall Destruction
}

Byung Gun Lim, MD, PhD, Jea Yeun Lee, MD, Mi Kyoung Lee, MD, PhD, Dong Kyu Lee, MD, PhD, Jun Suk Kim, MD, PhD, and Sang Sik Choi, MD, PhD

From: Department of Anesthesiology and Pain Medicine at Korea University Guro Hospital, South Korea

Dr. Lim is Clinical Assistant Professor with the Dept. of Anesthesiology and Pain Medicine, Korea University Medical Center, Guro Hospital, Seoul, Korea Dr. Choi is Associate Professor, Dept. of Anesthesiology and Pain Medicine, Korea University Medical Center, Guro Hospital, Seoul, Korea

Dr. JY Lee is Chief Resident, Dept. of Anesthesiology and Pain Medicine, Korea University Medical Center, Guro Hospital, Seoul, Korea

Dr. MK Lee is Professor, Dept. of Anesthesiology and Pain Medicine, Korea University Medical Center, Guro Hospital, Seoul, Korea

Dr. DK Lee is Clinical Assistant Professor, Dept. of Anesthesiology and Pain Medicine, Korea University Medical

Center, Guro Hospital, Seoul, Korea

Dr. Kim is Professor, Dept. of Medical

Oncology and Hematology, Korea

University Guro Hospital,, Seoul, Korea

Address correspondence:

Sang Sik Choi, MD, PhD

Associate Professor

Dept. of Anesthesiology and Pain Medicine

Korea University Guro Hospital 97, Gurodong-Gil, Guro-Gu Seoul 152-703, Kore

E-mail address: clonidine@empal.com

Disclaimer: This case report was funded by the Department of Anesthesiology and Pain Medicine at Korea University Guro Hospital.

Conflict of interest: None.

Manuscript received: 07/06/2011 Revised manuscript received: 08/03/2011 Accepted for publication: $08 / 23 / 2011$

Free full manuscript: www.pain physicianjournal.com
Background: Percutaneous balloon kyphoplasty is an effective, minimally invasive procedure that is used to relieve pain and stabilize spine fractures caused by severe osteoporosis or osteolysis due to tumor metastasis. However, there remains a risk of bone cement leakage during and after kyphoplasty, especially in cases with severe vertebral wall destruction or neurological deficits.

Objective: This article presents a case in which kyphoplasty was used to manage these complications in a woman with vertebral compression fractures caused by tumor metastasis.

Design: Case report.

Setting: Pain management clinic.

Methods: The patient was a 76-year-old woman who had severe low back pain, lower extremity weakness, and cauda equina syndrome because of vertebral compression fracture and spinal metastasis with epidural involvement. The patient had a large bony defect in the vertebra that the bone filler device could pass freely through the anterior body wall. Nevertheless, kyphoplasty was successfully performed by using our new cement injection technique, which is a slow injection of the highly viscous bone cement, followed by a second injection 10 minutes later to allow the previously injected cement to harden.

Results: The procedure significantly alleviated all symptoms. The day after the procedure, in the absence of additional pain medication, the pain had dropped dramatically to a numerical rating scale 3-4, and there was an improvement in motor function that allowed the patient to sit and go to the bathroom by herself. In addition, the voiding sensation had returned, which allowed the patient to defecate and urinate normally.

Limitations: This report describes a single case report.

Conclusion: Our new cement injection technique may allow balloon kyphoplasty to be safely and effectively performed in cancer patients with pathological vertebral compression fractures, even if there are large defects in the anterior vertebral wall and neurological deficits.

Key words: Compression fractures, kyphoplasty, metastasis, neurological deficits, osteolysis, polymethylmethacrylate.

Pain Physician 2011; 14:539-544 
P athological vertebral compression fracture (VCF) due to spinal metastasis can cause severe complications, including intractable pain, deformities of the vertebral bodies, and neurological complications, and is therefore associated with increased morbidity and mortality. Percutaneous balloon kyphoplasty is an effective, minimally invasive procedure that is used to relieve pain and stabilize spine fractures caused by severe osteoporosis or tumor metastasis (1-4).

However, there remains a risk of bone cement leakage during and after kyphoplasty, especially in cases characterized by vertebral wall destruction. Reported here is the case of a 76-year-old immobilized woman with intractable pain and severe neurological deficits due to VCF caused by the spinal metastasis of advanced hilar cholangiocarcinoma. The patient had a bony defect in the vertebra that was so large the bone filler device could pass freely through the anterior body wall. Despite this, kyphoplasty was successfully performed by using our new technique.

\section{Case Report}

A 76-year-old, $146 \mathrm{~cm}, 40 \mathrm{~kg}$ woman was referred to our pain clinic for her progressively worsening back pain over the previous 2 months. The pain was deep aching and aggravated by motion. The intensity of the pain was initially 4-5 on the 11-point numerical rating scale (NRS, $0=$ no pain and $10=$ worst possible pain) but progressively worsened to NRS 8-9 over a few days. The pain was so severe that the patient could not even move from her bed. She also had neurological symptoms, including lower extremity weakness and loss of urinary voiding sensation.

Her medical history included a subtotal gastrectomy 9 years ago for gastric cancer and a hilar cholangiocarcinoma that was diagnosed 6 months previously. Surgical removal of the tumor was not possible due to poor general condition of the patient and the presence of multiple metastases and poorly controlled asthma. Palpation over the spinous process of the T12, L1 vertebrae elicited severe pain. Sensory, motor and rectal examination revealed numbness in the saddle area, decreased muscle power (motor grade 3-4) of the legs and decreased anal tone and voluntary contraction, respectively. Plain radiographs ( $T-L$ spine) and magnetic resonance imaging (MRI) showed compression fractures with fluid-filled clefts in the T12, L1 vertebra and retropulsion of metastatic mass into the epidural space with compression of the conus medullaris at the level of the T12-L1 spine (Fig. 1).
Conservative treatments with analgesic medications, including strong opioid analgesics, radiotherapy, and chemotherapy failed to alleviate the pain. After discussion with the radiology department, we reached the conclusion that the posterior longitudinal ligament (PLL) was intact judging from the silhouette of the adjacent structures on serial MRI images. Therefore, kyphoplasty was performed. Prophylactic intravenous antibiotic (1 $\mathrm{g}$ of cefazolin) was administered before the procedure and the patient was placed in the prone position. Her blood pressure, heart rate, and pulse oximetry were monitored continuously. A total of $100 \mu \mathrm{g}$ of fentanyl was given intermittently as needed for pain control during the procedure. Kyphoplasty was performed with a bipedicular approach under C-arm fluoroscopic guidance. The bony defect was so large that when the bone filler device was advanced into the T12 vertebral body it could pass freely through the anterior body wall (Fig. 2A). Even though the size of the defect meant that there was a substantial risk that the cement would leak from the vertebral body, we decided to complete the procedure by using a new technique that involved 2 successive slow injections of highly viscous bone cement. After creating a void by ballooning (Fig. 2B), a small volume of bone cement was injected just posterior to the bony defect to build a barrier. This was performed under real-time C-arm fluoroscopic guidance. The injected bone cement was thicker than usual to minimize the risk of accidental leakage. After waiting for about 10 minutes to allow the injected cement to harden (Fig. 2C), a second aliquot of bone cement was injected into the vertebral body. The entire vertebral body was filled without leakage through the defect in the anterior wall of the vertebral body. In total, $4 \mathrm{~mL}$ of cement was injected on each side (Figs. 2D, 2E). The same method was used to fill the L1 vertebra with 6 $\mathrm{mL}$ of cement on each side. The post-procedural course was uneventful.

The procedure significantly alleviated all symptoms. The day after the procedure, in the absence of additional pain medication, the pain had dropped dramatically to NRS 3-4, and there was an improvement in motor function that allowed the patient to sit and go to the bathroom by herself. In addition, the voiding sensation had returned, which allowed the patient to defecate and urinate normally. A follow-up spine MRI revealed an impressive reduction in kyphotic angulation and cord compression, and cement leakage was not observed (Fig. 3). All of these symptomatic improvements were maintained for 6 months. 


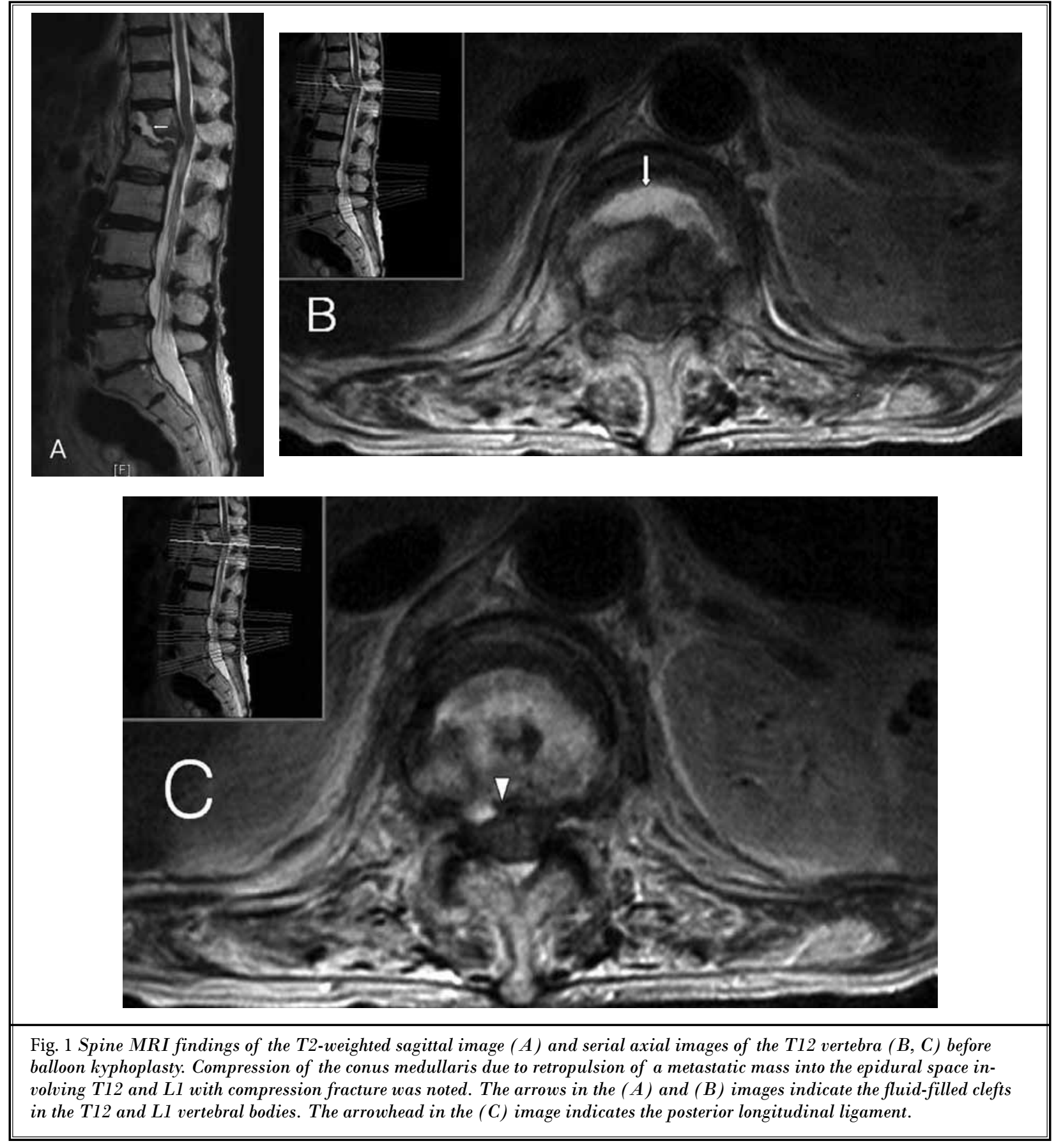

\section{Discussion}

The spine is the most common site for skeletal metastasis (5). Autopsy studies have shown that spinal metastasis occurs in 5-30\% of patients with cancer and that metastatic epidural spinal cord compression eventually affects about $20 \%$ of these patients (6-8). The osteolytic
VCF caused by spinal metastasis can result in various complications, including severe pain, kyphotic deformity of the vertebral body, instability, and neurological complications. All of these complications are associated with increased mortality and morbidity. 

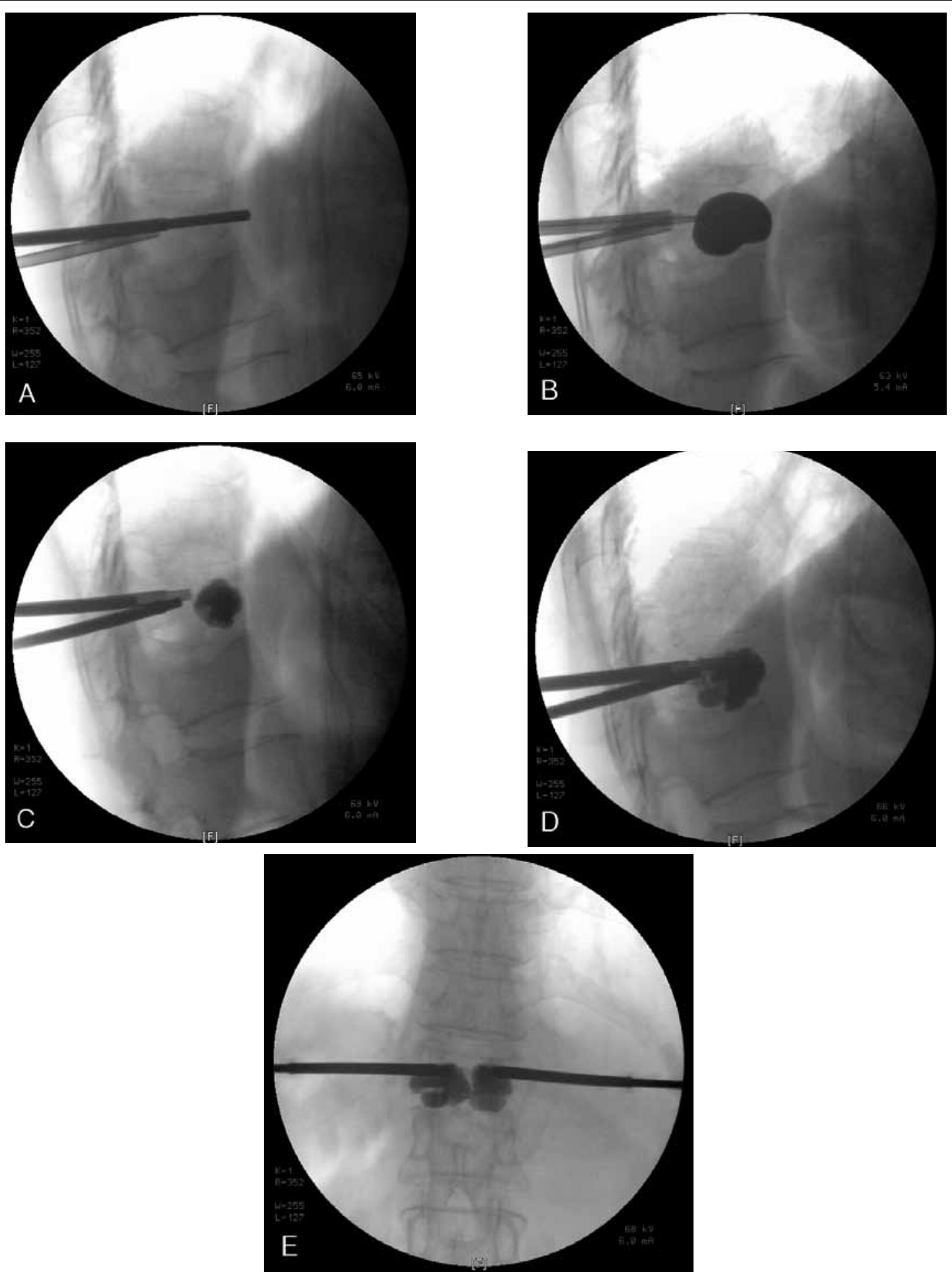

Fig. 2. Fluoroscopic images during balloon kyphoplasty on the T12 vertebra. When the bone filler device was advanced into the T12 vertebral body it could pass freely through the bony defect of the anterior body wall (A). A lateral image of the inflated balloon tamps in the anterior two-thirds of the fractured vertebral body $(B)$. A small volume of bone cement was injected just posterior to the bony defect to build a barrier $(C)$. Lateral (D) and anterior-posterior $(E)$ images of the second aliquot of bone cement that filled the cavity in the fractured vertebral body, respectively. Cement leakage was not detected. 


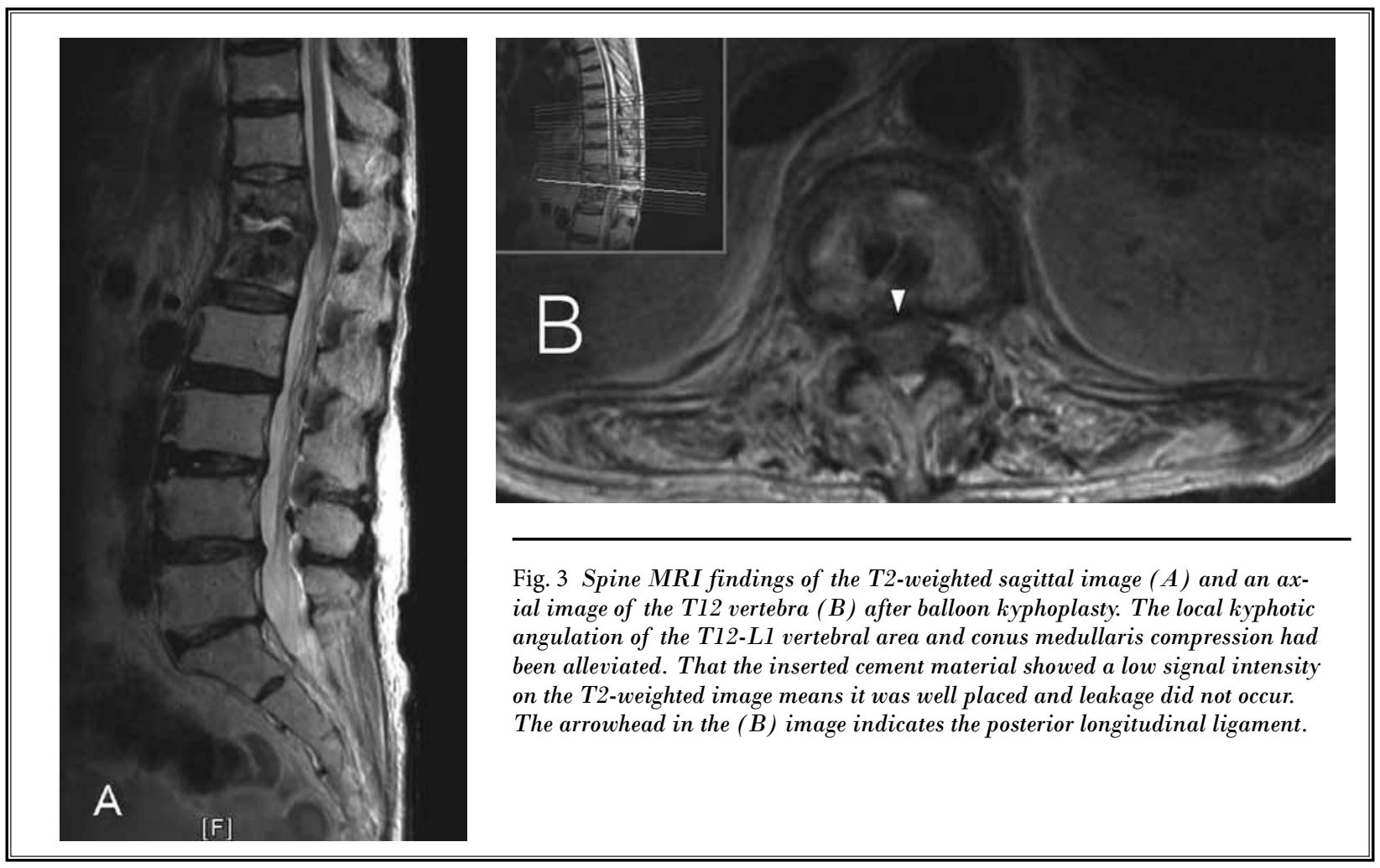

Various treatment options are available for VCF caused by spinal metastases, most of which are only palliative and aim to improve the quality of life. They include bed rest, bracing, physical therapy, medication, chemotherapy, radiotherapy, and interventional treatments. The latter include percutaneous vertebral augmentation therapies such as vertebroplasty and kyphoplasty, which are more effective and act more quickly than the other treatments and can be employed in patients with poor general condition.

Although kyphoplasty is a safe procedure that is associated with a lower risk of cement leakage than vertebroplasty, bone cement leakage can also occur during and after kyphoplasty, especially when, like in our patient, there is an osteolytic compression fracture with vertebral wall destruction. There are previous reports that while the rate of balloon kyphoplasty-associated cement leakage is negligible in patients with compression fractures that result from osteoporosis (9), it is as high as $12.1-12.5 \%$ in patients with metastatic spinal tumors (10). Moreover, if there is a cavity inside a vertebral body, the probability of cement leakage is very high (11). In the case reported here, the chance of cement leakage was very high because of the size of the bony defect. However, there was no choice but to perform kyphoplasty. The patient had been treated with and eventually failed with various treatments, including radiotherapy and chemotherapy, to alleviate the symptoms, and was considered to be inoperable because of her poor general condition. A new cement injection technique was devised to minimize the chance of leakage.

The cement used was polymethylmethacrylate (PMMA) bone cement (Surgical Simplex30, Howmedica International Inc., Clare, Ireland), which was prepared by mixing PMMA powder and liquid monomer. When the PMMA powder and liquid monomer are mixed, exothermic polymerization occurs, and the runny liquid mixture changes into a dough-like material over time. This is then inserted into the cancellous bone space. The polymerization of the bone cement occurs over a period of 3 to 10 minutes. When Belkoff et al (12) measured the internal vertebral body temperature during polymerization, they found that polymerization is complete within 8 minutes of the bone cement injection into the vertebral body.

These characteristics of PMMA bone cement allowed us to devise a new cement injection technique. This technique employed 2 successive injections of highly viscous bone cement separated by 10 minutes. The 
first injection, where a small amount of bone cement was injected just posterior to the bony defect, was used to make a kind of cement wall. This was succeeded by a pause of 10 minutes to allow the injected cement to harden completely. A second injection was then used to fill the vertebral space. This kyphoplasty method was both successful and safe.

There has been a report of a bone fragment being displaced while inflating the balloon during kyphoplasty (13). If a fracture in the posterior surface of the vertebral body or cauda equina syndrome due to spinal cord compression exists, inflating the balloon excessively carries the possibility of a fractured segment being displaced to the posterior direction and causing neurologic injury. So, it would be safe to inflate the balloon minimally to secure space just big enough for cement injection, and try not to restore the vertebral height.

But in this case, the compression fracture generated a backward force which resulted in a segment displacement into the epidural space and caused spinal cord compression. Therefore, sufficient restoration of the vertebral body height would counterbalance the back- ward force, which would induce decompression of the spinal cord, so we carefully and sufficiently performed ballooning to restore the vertebral body height. As expected, the patient's neurological symptoms, including lower extremity weakness and the loss of voiding sensation, were dramatically improved. Moreover, of critical importance to the success and safety of this procedure was the fact that the PLL was intact because this can allow for decompression of the cauda equina as height was restored. It can also prevent cement leakage to the spinal cord and nerves. In this case, the procedure was carried out after confirming with the pretreatment MRI that the PLL was intact (Fig. 1C), and afterwards, it was verified with the post-treatment MRI (Fig. 3B).

\section{Conclusion}

The described cement injection technique allows balloon kyphoplasty to be safely and effectively performed in cancer patients with pathological VCFs, even if there are large defects in the anterior vertebral wall and neurological deficits.

\section{RefERENCES}

1. Hirsch AE, Jha RM, Yoo AJ, Saxena A, Ozonoff A, Growney MJ, Hirsch JA. The use of vertebral augmentation and external beam radiation therapy in the multimodal management of malignant vertebral compression fractures. Pain Physician 2011; 14:447-458.

2. Powell MF, DiNobile D, Reddy AS. Carm fluoroscopic cone beam CT for guidance of minimally invasive spine interventions. Pain Physician 2010; 13:5159.

3. Jones JO, Bruel BM, Vattam SR. Management of painful vertebral hemangiomas with kyphoplasty: A report of two cases and a literature review. Pain Physician 2009; 12:E297-E303.

4. Gill JB, Kuper M, Chin PC, Zhang Y, Schutt R. Comparing pain reduction following kyphoplasty and vertebroplasty for osteoporotic vertebral compression fracturs. Pain Physician 2007; 10:583590.
5. Aaron AD. The management of cancer metastatic to bone. JAMA 1994; 272:12061209.

6. Siegal T. Surgical decompression of anterior and posterior malignant epidural tumors compressing the spinal cord: A prospective study. Neurosurgery 1985; 17:424-432.

7. Sundaresan N, Digiacinto GV, Hughes JE. Surgical treatment of spinal metastases. Clin Neurosurg 1986; 33:503-522.

8. Wong DA, Fornasier VL, MacNab I. Spinal metastases: The obvious, the occult, and the impostors. Spine (Phila Pa 1976) 1990; 15:1-4.

9. De Negri P, Tirri T, Paternoster G, Modano $P$. Treatment of painful osteoporotic or traumatic vertebral compression fractures by percutaneous vertebral augmentation procedures: A nonrandomized comparison between vertebroplasty and kyphoplasty. Clin J Pain 2007; 23:425430 .
10. Pflugmacher R, Beth P, Schroeder RJ, Schaser KD, Melcher I. Balloon kyphoplasty for the treatment of pathological fractures in the thoracic and lumbar spine caused by metastasis: One-year follow-up. Acta Radiol 2007; 48:89-95.

11. Peh WC, Gelbart MS, Gilula LA, Peck DD. Percutaneous vertebroplasty: Treatment of painful vertebral compression fractures with intraosseous vacuum phenomena. AJR Am J Roentgenol 2003; 180:1411-1417.

12. Belkoff SM, Molloy S. Temperature measurement during polymerization of polymethylmethacrylate cement used for vertebroplasty. Spine (Phila Pa 1976) 2003; 28:1555-1559.

13. Dudeney S, Lieberman IH, Reinhardt MK, Hussein M. Kyphoplasty in the treatment of osteolytic vertebral compression fractures as a result of multiple myeloma. J Clin Oncol 2002; 20:23822387. 